\title{
The Sixth Order P-stable Method with Minimal Phase Lag for a Second Order Initial-value Problem
}

\author{
Kaili Xiang \\ Depart. of Applied Math., Southwestern Univ. of Finance and Economics, Chengdu 610074, China
}

\begin{abstract}
Several symmetric (hybrid) two step sixth-order Pstable methods for the numerical integration of second order periodic initial value problems have been considered in this paper. These methods have minimal phase-lag than some sixth order methods in [1]-[5]. The theoretical and numerical results show that these methods are more efficient than those proposed in [1]-[5].
\end{abstract}

Keywords: Second order equation, Periodic initial value problems, P-stable, interval of periodicity, Phase-lag.

\section{Introduction}

We consider a class of symmetric (hybrid) two step methods presented in [1] for solving the second order initial value problem

$$
y^{\prime \prime}=f(t, y), y\left(t_{0}\right)=y_{0}, y^{\prime}\left(t_{0}\right)=y_{0}^{\prime},
$$

which arises in the theory of orbital mechanics and in the study of wave equations.

It is known that the Numerov's method is the most popular technique, which has fourth order phase-lag and possesses as only a finite interval of periodicity $\left(0,2.449^{2}\right)$. Cash [1], Chawla and Rao [2], Ananthakrishnaiah [3] developed sixth-order Pstable method with sixth order phase-lag. Thomas [4] developed sixth order almost P-stable formulae with eighth order phase lag. Xiang Kaili et.[5] proposed a class sixth order P-stable or almost P-stable method with sixth order phase lag.

The purpose of this paper is, by modifying the methods in [1]-[5] and selecting parameters suitably, to obtain a family sixth order P-stable methods for $M_{6}\left(\hat{A}, \alpha, \beta_{1}\right)$ which are P-stable and with $(2 m+4)$ order phase-lag. It is shown that the sixth order P-stable methods have minimal phase lag comparing to those presented in [1]-[5]. The implementation of these methods in this paper and numerical illustration on one simple problem will be analyzed in detail.

\section{Basic theory}

We apply the symmetry implicit two-step method to the test equation

$$
y^{\prime \prime}=-\lambda^{2} y, \lambda>0,
$$

which has the stability polynomial

$$
\Omega\left(\xi, H^{2}\right)=A(H) \xi^{2}-2 B(H) \xi+A(H), H=\lambda h,
$$

where $A(H)$ and $B(H)$ are polynomials of $H=\lambda h$. We call $\Omega\left(\xi, H^{2}\right)=0$ as the characteristic equation.

Definition 1.(Lambert and Watson [6]) The method with stability polynomial (3) is said to have interval of periodicity $\left(0, H_{p}^{2}\right)$ if for all $H^{2}=$ $\left(0, H_{p}^{2}\right)$, the roots $\xi_{1,2}$ of $\Omega\left(\xi, H^{2}\right)$ satisfy

$$
\xi_{1,2}=\exp ( \pm i \theta(H)),
$$

for some real valued function $\theta(H)$.

Definition 2. The method with stability polynomial (3) is said to be P-stable if its interval of periodicity is $(0, \infty)$. It is easy to see that the roots of (3) are complex and of module one if

$$
\left|\frac{B(H)}{A(H)}\right|<1
$$

Thus, the P-stability condition is valid if

$$
\begin{array}{r}
A(H)+B(H)>0, A(H)-B(H)>0, \\
\text { for all } H^{2} \in(0, \infty) .
\end{array}
$$

The exact solution of test equation (2) with the initial condition $y\left(t_{0}\right)=y_{0}$ and $y^{\prime}\left(t_{0}\right)=y_{0}^{\prime}$ is given by

$$
y(t)=y_{0} \cos \lambda t+\frac{y_{0}^{\prime}}{\lambda} \sin \lambda t .
$$

Evaluating (7) at $t_{n+1}, t_{n}, t_{n-1}$ and eliminating $y_{0}, y_{0}^{\prime}$, we obtain

$$
y\left(t_{n+1}\right)-2 \cos (\lambda h) y\left(t_{n}\right)+y\left(t_{n-1}\right)=0,
$$


whose characteristic equation is

$$
\xi^{2}-2 \cos \theta(H) \xi+1=0, H=\lambda h .
$$

Therefore, the characteristic equation of (3) is

$$
\xi^{2}-2 \cos \theta(H) \xi+1=0,
$$

where $\cos \theta(H)=\frac{B(H)}{A(H)}$.

Definition 3. (Ananthakrishnaiah [3]) We define the phase lag of the method with stability polynomial (3) as the leading term in the expansion of

$$
\frac{A(H) \cos (H)-B(H)}{H^{2}}, \text { denoted by } P(H) .
$$

\section{A family of sixth-order P- stable methods with mini- mal phase-lag}

For the numerical integration of second-order periodic initial value problem (1), we consider a family of implicit two-step sixth-order methods

$$
y_{n}^{[i]}=y_{n}-\beta_{i} h^{2}\left(f_{n+1}-2 f_{n}^{[i-1]}+f_{n-1}\right), f_{n}^{[0]}=f_{n},
$$

where $i=1,2, \ldots, m$.

$$
\begin{aligned}
y_{n+\alpha} & =\hat{A} y_{n+1}+(1+\alpha-2 \hat{A}) y_{n}+(\hat{A}-\alpha) y_{n-1} \\
& +h^{2}\left\{\left(\frac{\alpha^{4}}{24}+\frac{\alpha^{3}}{12}-\frac{\hat{A}}{12}-\frac{\alpha}{12}\right) f_{n+1}\right. \\
& +\left(-\frac{\alpha^{4}}{12}+\frac{\alpha^{2}}{2}-\frac{5 \hat{A}}{6}+\frac{5 \alpha}{12}\right) f_{n}^{[m]} \\
& \left.+\left(\frac{\alpha^{4}}{24}-\frac{\alpha^{3}}{12}-\frac{\hat{A}}{12}+\frac{\alpha}{8}\right) f_{n-1}\right\} . \\
& =\tilde{A} y_{n+1}+(1-\alpha-2 \tilde{A}) y_{n}+(\tilde{A}+\alpha) y_{n-1} \\
y_{n-\alpha} & h^{2}\left\{\left(\frac{\alpha^{4}}{24}-\frac{\alpha^{3}}{12}-\frac{\tilde{A}}{12}+\frac{\alpha}{12}\right) f_{n+1}\right. \\
& +\left(-\frac{\alpha^{4}}{12}+\frac{\alpha^{2}}{2}-\frac{5 \tilde{A}}{6}-\frac{5 \alpha}{12}\right) f_{n}^{[m]} \\
& \left.+\left(\frac{\alpha^{4}}{24}-\frac{\alpha^{3}}{12}-\frac{\tilde{A}}{12}-\frac{\alpha}{8}\right) f_{n-1}\right\} .
\end{aligned}
$$

Thus, for $n \geqslant 1$, the $(m+2)$-parameter family of sixth-order method for $y^{\prime \prime}=f(t, y)$ is given by

$$
\begin{aligned}
y_{n+1}-2 y_{n}+y_{n-1} & =h^{2}\left\{\left(\frac{1}{12}-\frac{1}{20\left(1-\alpha^{2}\right)}\right)\right. \\
& \times\left(f_{n+1}+f_{n-1}\right) \\
& +\left(\frac{5}{6}-\frac{1}{10 \alpha^{2}}\right) f_{n} \\
& +\frac{1}{20 \alpha^{2}\left(1-\alpha^{2}\right)} \\
& \left.\times\left(f_{n+\alpha}+f_{n-\alpha}\right)\right\}
\end{aligned}
$$

where $0<\alpha<1, \hat{A}+\tilde{A}=\alpha^{2}, f_{n}^{[i]}=f\left(t_{n}, y_{n}^{[i]}\right)$, $i=1,2, \ldots, m, f_{n \pm 1}=f\left(t_{n \pm 1}, y_{n \pm 1}\right), f_{n \pm \alpha}=$ $f\left(t_{n \pm \alpha}, y_{n \pm \alpha}\right)$ and $\beta_{i}(i=1,2, \ldots, m), \alpha$ and $\hat{A}$ are free parameters.

If we apply the method (15) to test equation (2), we have the stability polynomial

$$
\Omega\left(\xi, H^{2}\right)=A(H) \xi^{2}-2 B(H) \xi+A(H),
$$

where

$$
\begin{aligned}
A(H) & =1+\frac{H^{2}}{12}+\frac{H^{4}}{240}-\frac{1}{120} \sum_{k=1}^{m}\left[(-1)^{k+1}\right. \\
& \left.\times 2^{k-1} \prod_{l=1}^{k} \beta_{m-l+1}\right] H^{2 k+4} \\
B(H) & =1-\frac{5 H^{2}}{12}+\frac{H^{4}}{240}-\frac{1}{120} \sum_{k=1}^{m}\left[(-1)^{k+1}\right. \\
& \left.\times \quad 2^{k-1} \prod_{l=1}^{k} \beta_{m-l+1}\right] H^{2 k+4}
\end{aligned}
$$

Thus

$$
\begin{gathered}
A(H)-B(H)=\frac{H^{2}}{2}>0, \\
\text { for all } H^{2} \in(0, \infty), \\
A(H)+B(H)=2-\frac{H^{2}}{3}+\frac{H^{4}}{120} \\
-\frac{1}{60} \sum_{k=1}^{m}\left[(-1)^{k+1} 2^{k-1} \prod_{l=1}^{k} \beta_{m-l+1}\right] H^{2 k+4} .
\end{gathered}
$$

From (17), (18) and Definition 3, we have

$$
\begin{aligned}
\frac{A(H) \cos (H)-B(H)}{H^{2}} & =\sum_{k=2}^{m+1}(-1)^{k}\left[\frac{1}{(2 k+4) !}\right. \\
& -\frac{1}{12(2 k+2) !}+\frac{1}{240(2 k) !} \\
& +\frac{1}{120} \sum_{l=1}^{k-1}\left(\frac{2^{l-1}}{(2(k-l)) !}\right. \\
& \left.\left.\times \prod_{r=1}^{l} \beta_{m-r+1}\right)\right] H^{2 k+2} \\
& +O\left(H^{2 m+6}\right),
\end{aligned}
$$

It follows (21) and Definition 3 that

Theorem 1. If the parameters $\beta_{2}, \beta_{3}, \ldots, \beta_{m}$ 
appearing in (12) are given by

$$
\begin{aligned}
\beta_{m} & =-\frac{5}{252}, \quad m \geqslant 2, \\
\beta_{m-k+2} & =-\frac{120}{2^{k-3} \prod_{\gamma=0}^{k-3} \beta_{m-\gamma}}\left[\frac{1}{(2 k+4) !}\right. \\
& -\frac{1}{12(2 k+2) !}+\frac{1}{240(2 k) !} \\
& +\frac{1}{120} \sum_{l=1}^{k-2}\left(\frac{2^{l-1}}{(2(k-l)) !}\right. \\
& \left.\left.\times \prod_{r=1}^{l} \beta_{m-r+1}\right)\right], \quad k=3, \ldots, m,
\end{aligned}
$$

then, the sixth-order implicit method (15) has the phase lag of

$$
\begin{aligned}
P(H) & =\left\{\frac{1}{(2 m+6) !}-\frac{1}{12(2 m+4) !}\right. \\
& +\frac{1}{240} \frac{1}{(2 m+2) !} \\
& +\frac{1}{120} \sum_{l=1}^{m}\left(\frac{2^{l-1}}{(2(m-l+1)) !}\right. \\
& \left.\left.\times \prod_{r=1}^{l} \beta_{m-r+1}\right)\right\} H^{2 m+4} .
\end{aligned}
$$

Therefore, if $\beta_{2}, \beta_{3}, \ldots, \beta_{m}$ are given by (22), we obtained three-parameters $\hat{A}, \alpha, \beta_{1}$ family of sixthorder implicit method (15) with minimal phase-lag of order $2 m+4$, which is denoted as $M_{6}\left(\hat{A}, \alpha, \beta_{1}\right)$.

Selecting parameter $\beta_{1}$ of method $M_{6}\left(\hat{A}, \alpha, \beta_{1}\right)$ suitably, we have

$$
A(H)+B(H)>0 \text { for all } H^{2} \in(0, \infty) .
$$

From (19), (20) and (6), a family of sixth-order P-stable methods $M_{6}\left(\hat{A}, \alpha, \beta_{1}\right)$ with $2 m+4$ order phase-lag can be obtained by suitably selecting parameter $\beta_{1}$ as following table 1 .

Taking $m=2, \alpha=\frac{1}{2}, \hat{A}=\frac{3}{8}$, a particular sixth order method of this class is given by $I M_{6}\left(\beta_{1}\right)$

$$
\begin{aligned}
\bar{y}_{n}=y_{n}-\beta_{1} h^{2}\left(f_{n+1}-2 f_{n}+f_{n-1}\right), \\
\hat{y}_{n}=y_{n}+\frac{5}{252} h^{2}\left(f_{n+1}-2 \bar{f}_{n}+f_{n-1}\right), \\
y_{n+\frac{1}{2}}=\frac{3}{8} y_{n+1}+\frac{3}{4} y_{n}-\frac{1}{8} y_{n-1} \\
-\frac{1}{128} h^{2}\left(5 f_{n+1}-2 \hat{f}_{n}-3 f_{n-1}\right),
\end{aligned}
$$

\begin{tabular}{|l|l|l|}
\hline $\mathrm{m}$ & 1 & 2 \\
\hline Methods & Methods 1 & Methods 2 \\
\hline$\beta_{1}$ & $-\frac{13+2 \sqrt{55}}{972}$ & $\begin{array}{l}<-2.560009 E- \\
02\end{array}$ \\
\hline$\beta_{2}$ & & $-\frac{5}{252}$ \\
\hline$\beta_{3}$ & & \\
\hline$\beta_{4}$ & & \\
\hline $\begin{array}{l}\text { Phase- } \\
\text { lag }\end{array}$ & $\frac{2\left(5+252 \beta_{1}\right)}{3 \times 8 !} H^{6}$ & $-\frac{3\left(7+400 \beta_{1}\right)}{2 \times 10 !} H^{8}$ \\
$\mathrm{P}(\mathrm{H})$ & & \\
\hline $\mathrm{m}$ & 3 & 4 \\
\hline Methods & Methods 3 & Methods 4 \\
\hline$\beta_{1}$ & $<0$ & $<-2.187734 E-$ \\
\hline$\beta_{2}$ & $-\frac{7}{400}$ & $-\frac{5}{308}$ \\
\hline$\beta_{3}$ & $-\frac{5}{252}$ & $-\frac{7}{400}$ \\
\hline$\beta_{4}$ & & $-\frac{5}{252}$ \\
\hline $\begin{array}{l}\text { Phase- } \\
\text { lag } \\
\mathrm{P}(\mathrm{H})\end{array}$ & $\frac{9\left(5+308 \beta_{1}\right)}{12 !} H^{10}$ & $-\frac{7061+491400 \beta_{1}}{30 \times 14 !} H^{12}$ \\
\hline
\end{tabular}

Table 1: P-stable sixth-order methods $M_{6}\left(\hat{A}, \alpha, \beta_{1}\right)$ with phase-lag of order $2 m+4$

$$
\begin{aligned}
y_{n-\frac{1}{2}} & =-\frac{1}{8} y_{n+1}+\frac{3}{4} y_{n}+\frac{3}{8} y_{n-1} \\
& -\frac{1}{128} h^{2}\left(-f_{n+1}-2 \hat{f}_{n}+5 f_{n-1}\right), \\
y_{n+1}-2 y_{n}+y_{n-1} & =\frac{1}{60} h^{2}\left\{\left(f_{n+1}+f_{n-1}\right)\right. \\
& \left.+26 f_{n}+16\left(y_{n+\frac{1}{2}}+y_{n-\frac{1}{2}}\right)\right\},
\end{aligned}
$$

where

$$
\begin{aligned}
& f_{n \pm 1}=f\left(t_{n \pm 1}, y_{n \pm 1}\right), \quad f_{n \pm \frac{1}{2}}=f\left(t_{n \pm \frac{1}{2}}, y_{n \pm \frac{1}{2}}\right), \\
& \bar{f}_{n}=f\left(t_{n}, \bar{y}_{n}\right), \quad \hat{f}_{n}=f\left(t_{n}, \hat{y}_{n}\right) .
\end{aligned}
$$

We knows, when $\beta_{1}<-2.560009 E-02$, the sixth order $I M_{6}\left(\beta_{1}\right)$ is $\mathrm{P}$-stable, and the eighth order phase-lag

$$
P(H)=-\frac{7+400 \beta_{1}}{2419200} H^{8} .
$$

Cash used the sixth order P-stable method (see [1]) to obtain the phase lag $P(H)=-000001378 H^{6}$ while the sixth order method with eighth order phase lag has been given by Thomas [4] to show the interval of periodicity $(0,7.68)$. Xiang Kaili. et. [5] have given a family of sixth order methods with sixth order phase-lag.

From Table 1, we conclude that new sixth-order P-stable method has smaller phase-lag than the same order implicit methods in [1]-[5]. 


\section{Numerical illustration}

We note that for non-linear $f(t, y)$ all these methods are implicit and need an iterative process for computing the solution at each step. To arrive at this aim, we briefly consider the application of the modified Newton's method.

The sixth order $M_{6}\left(\hat{A}, \alpha, \beta_{1}\right)$ method is written by the form

$$
G\left(y_{n+1}\right)=y_{n+1}-\phi\left(y_{n+1}\right)=0 .
$$

Suppose that $y_{n+1}^{0}$ denote an initial approximation for $y_{n+1}$ defined by

$$
y_{n+1}^{0}=2 y_{n}-y_{n-1}+h^{2} f_{n} .
$$

We obtain that the modified Newton's method for (24) is

$$
\begin{gathered}
G\left(y_{n+1}^{(i)}\right)+G\left(y_{n+1}^{\prime(0)}\right) \Delta y_{n+1}^{(i)}, y_{n+1}^{(i+1)}=0 \\
=y_{n+1}^{(i)}+\triangle y_{n+1}^{(i)}, i=0,1,2, \cdots,
\end{gathered}
$$

which converges for sufficiently small $h$. Indeed, the convergence can be derived by using $\left|y_{n+1}^{(0)}-y_{n+1}\right|=$ $O\left(h^{4}\right)$ and $\left|1-G^{\prime}\left(y_{n+1}^{(0)}\right)\right|=O\left(h^{2}\right)$, and continuous property of $G^{\prime}(y)$.

Example 1.(Lambert and Watson [6]) Problem

$Z^{\prime \prime}+Z=0.001 \exp (i t), Z(0)=1, Z^{\prime}(0)=0.9995 i$,

has exact solution $Z(t)=\exp (i t)(1-0.0005 i t)$. We computed $\gamma(t)=\sqrt{\mu(t)^{2}+\nu(t)^{2}}$, where $Z(t)=$ $\mu(t)+\nu(t)$ and $t=40 \pi$, by the $I M_{6}\left(\beta_{1}\right)\left(\beta_{1}=\right.$ $-0.03)$, Cash's sixth order methods [1], Chawla and Rao's sixth order method [2] and Xiang kaili's sixth order P-stable methods [5].

In Table 2, we shall give the absolute errors in $\gamma(40 \pi)$ by using $h=\frac{\pi}{4}, \frac{\pi}{5}, \frac{\pi}{6}, \frac{\pi}{9}, \frac{\pi}{12}$. It will be seen that the sixth order method $I M_{6}\left(\beta_{1}\right)$ is more efficient than the sixth order formulas presented by Cash [1], Chawla and Rao [2] and Xiang kaili [5].

\section{Acknowledgment}

This work was supported by the Scientific Research Fund of Southwestern University of Finance and Economics(Grant No. 07YB26). The author's email address: xiangkl@swufe.edu.cn

\section{References}

[1] J.R. Cash, High order P-stable formulae for the numerical integration of periodic initial value problems, Numer. Math., 37: 335-370, 1981.

\begin{tabular}{|c|c|}
\hline $\mathrm{h}$ & Cash's 6th order method \\
\hline$\frac{\pi}{4}$ & $1.25 \times 10^{-5}$ \\
\hline$\frac{\pi}{5}$ & $3.39 \times 10^{-6}$ \\
\hline$\frac{\pi}{6}$ & $1.19 \times 10^{-6}$ \\
\hline$\frac{\pi}{9}$ & $9.79 \times 10^{-8}$ \\
\hline$\frac{\pi}{12}$ & $1.71 \times 10^{-8}$ \\
\hline $\mathrm{h}$ & Chawla and Rao' method $M_{6}(0)$ \\
\hline$\frac{\pi}{4}$ & $1.08 \times 10^{-3}$ \\
\hline$\frac{\pi}{5}$ & $2.74 \times 10^{-4}$ \\
\hline$\frac{\pi}{6}$ & $5.27 \times 10^{-5}$ \\
\hline$\frac{\pi}{9}$ & $3.82 \times 10^{-7}$ \\
\hline$\frac{\pi}{12}$ & $1.22 \times 10^{-4}$ \\
\hline $\mathrm{h}$ & Xiang Kaili.et'6th order method \\
\hline$\frac{\pi}{4}$ & $1.68 \times 10^{-6}$ \\
\hline$\frac{\pi}{5}$ & $7.29 \times 10^{-7}$ \\
\hline$\frac{\pi}{6}$ & $6.28 \times 10^{-8}$ \\
\hline$\frac{\pi}{9}$ & $4.25 \times 10^{-9}$ \\
\hline$\frac{\pi}{12}$ & $1.32 \times 10^{-4}$ \\
\hline$h$ & $1.56 \times 10^{-6}$ \\
\hline$\frac{\pi}{4}$ & $6.61 \times{ }^{1} 0-7$ \\
\hline$\frac{\pi}{5}$ & $5.23 \times 10^{-8}$ \\
\hline$\frac{\pi}{6}$ & $2.34 \times 10^{-9}$ \\
\hline$\frac{\pi}{9}$ & \\
\hline$\frac{\pi}{12}$ & $\left.\beta_{1}\right)\left(\beta_{1}=-0.03\right)$ \\
\hline & \\
\hline
\end{tabular}

Table 2: Absolute errors for different values of $h$ in $\gamma(40 \pi)$ of the problem $(27)$ 
[2] M.M. Chawla and P.S. Rao, High accuracy Pstable methods for $y^{\prime \prime}=f(t, y)$, IMA J. Numer. Analy., 5: 215-220, 1985.

[3] U. Ananthakrishnaiah, A class of two step Pstable methods for the accurate integration of second order periodic initial-value problems, $J$. Comput. Appl. Math., 14: 455-459, 1986.

[4] R.M. Thomas, Phase properties of high order, almost P-stable formulae, BIT, 24: 225-238, 1984.

[5] K.L. Xiang and J.X. Liu, High accuracy hybrid formula for $y^{\prime \prime}=f(t, y)$, ACTA Mathematicae Applicatae Sinica, 14(2): 218-221, 1998.

[6] J.D. Lambert and I.A. Watson, Symmetric multistep methods for periodic initial value problems, J.Inst.Maths. Appl., 18: 189-202, 1976.

[7] K.L. Xiang and R. M. Thomas, Efficient sixth order P-stable Methods with minimal local truncation error for $y^{\prime \prime}=f(t, y), J$. Comput. Math., 20(2): 175-184, 2002. 